\title{
The brain decade in debate: VII. Neurobiology of sleep and dreams
}

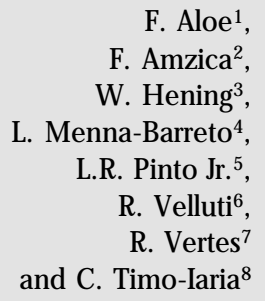

\author{
IInstituto de Psiquiatria, Faculdade de Medicina, Universidade de São Paulo, \\ São Paulo, SP, Brasil \\ ${ }^{2}$ Department of Physiology, School of Medicine, University of Laval, \\ Quebec City, Canada \\ ${ }^{3}$ Rutgers State U niversity, Center for Neuroscience, N ewark, NJ, USA \\ ${ }^{4}$ Departamento de Fisiologia e Biofísica, Instituto de Ciências Biomédicas, \\ Universidade de São Paulo, São Paulo, SP, Brasil \\ 5Instituto do Sonho, Universidade Federal de São Paulo, São Paulo, SP, Brasil \\ ${ }^{6}$ Neurofisiología, Departamento de Fisiología, Facultad de Medicina de Montevideo, \\ Montevideo, Uruguay \\ ${ }^{7}$ Center for Complex Systems, Florida Atlantic U niversity, Boca Raton, FL, USA \\ ${ }^{8}$ Departamento de Neurologia, Faculdade de Medicina, U niversidade de São Paulo, \\ São Paulo, SP, Brasil
}

\section{Correspondence \\ C. Timo-laria \\ Departamento de Neurologia \\ Faculdade de Medicina, USP \\ Av. Dr. Arnaldo, 455 \\ 01246-903 São Paulo, SP \\ Brasil \\ E-mail: cetiaria@usp.br}

The names of the authors are presented in alphabetical order, except for the last one that corresponds to the symposium organizer.

Publication supported by FAPESP.

Received August 8, 2001

Accepted September 4, 2001

\section{Abstract}

This article is a transcription of an electronic symposium held on February 5, 2001 by the Brazilian Society of Neuroscience and Behavior (SBNeC) during which eight specialists involved in clinical and experimental research on sleep and dreaming exposed their personal experience and theoretical points of view concerning these highly polemic subjects. Unlike most other bodily functions, sleep and dreaming cannot, so far, be defined in terms of definitive functions that play an ascribable role in maintaining the organism as a whole. Such difficulties appear quite clearly all along the discussions. In this symposium, concepts on sleep function range from a protective behavior to an essential function for maturation of the nervous system. Kleitman's hypothesis [Journal of Nervous and Mental Disease (1974), 159: 293-294] was discussed, according to which the basal state is not the wakeful state but sleep, from which we awake to eat, to protect ourselves, to procreate, etc. Dreams, on the other hand, were widely discussed, being considered either as an important step in consolidation of learning or simply the conscious identification of functional patterns derived from the configuration of released or revoked memorized information.

\section{Introduction}

Sleep and dreams have been a target for all kinds of myths and mystic explanations from remote antiquity to present times. Aristotle discovered that vertebrates and some invertebrate animals do sleep and was convinced that at least mammals and birds dream, a point of view that was shared in the nine-

\section{Key words}

- Sleep

- Dreams

- Consciousness

- Rapid eye movement

- REM teenth century by Darwin. Aristotle proved that bees sleep, a fact that was rediscovered in 1980 . He also discovered eye, lip, face and limb movements during dreaming and knew very well that such movements are related to dream content. In the nineteenth century several clinicians and physiologists confirmed the Aristotelian discoveries but unfortunately all that knowledge remained buried. 
Aserinsky and Kleitman (1), in 1953, started a new wave of discoveries that, in addition to confirming Aristotle, led to many recent advances as to fine behavioral and electrophysiological manifestations of sleep and dreaming, to the neural systems responsible for their generation, and to some cellular mechanisms involved in both functions. This article is an edited transcription of a virtual symposium promoted by the Brazilian Society of Neuroscience and Behavior (SBNeC) on February 5, 2001 in which several aspects of these subjects were discussed by clinicians and physiologists that do research on sleep and dreaming. Much remains to be discovered, however, as to the mechanisms of sleep and dreams and to their functions.

Cesar Timo-Iaria: Dear colleagues who joined us to discuss such an interesting subject, sleep and dreams, thank you for joining us in this virtual symposium. To begin our discussion I will ask you all two fundamental questions: 1) In your opinion what is (or are) the function(s) of sleep? and 2) What is a dream?

Luciano Ribeiro Pinto Jr.: Dreams are a state of consciousness different from wakefulness. Considering the first question, we could start by asking not why we sleep, but why we are awake.

Luiz Menna-Barreto: I see sleep as a protective behavior in its general aspect, and as an envelope of functions in its more particular aspects, such as hormone secretions and core temperature regulation.

Flavio Aloe: In what precise meaning would sleep be a protective behavior if the animal is more vulnerable while asleep?

Luiz Menna-Barreto: Sleep is protective in the sense that the prey becomes less conspicuous while sleeping, and coincides with the phase of the day/night cycle where the prey's sensorimotor equipment is below optimal performance.

Flavio Aloe: I believe there is no simple answer to either of your questions. Sleep serves a multitude of functions, ranging from CNS maturation to synaptic restoration, memory processes and many other functions.

Luiz Menna-Barreto: Dream is a name we give to describe one of the many possible states of consciousness.

Robert Vertes: I think that it is important to separate dreams and their function from sleep and its function. Obviously, dreams occupy a very small percentage of sleep, and are for the most part confined to rapid eye movement (REM) sleep which, in itself, represents about $20 \%$ of sleep. In addition, dreams do not become dreams unless they reach "dream consciousness", that is, we are aware of them. In this regard, it seems that the neurophysiological substrates for "potential" dreams are present throughout REM, but dreams only become dreams when they are recalled - which, even accounting for individual differences, occurs somewhat infrequently given the total amount of REM sleep.

Ricardo Velluti: Dreams are a consequence of at least three phenomena: 1) information stored in memory, 2) the incoming sensory input, and 3) the information that may come through our genetics.

Luciano Ribeiro Pinto Jr.: Jouvet's (2) hypothesis links dreams to the preservation of the genetic integrity of our personality.

Flavio Aloe: Do you mean the generation of dreams in general or of specific dream contents to unload psychological stress of any nature?

Ricardo A. Velluti: 1) To the best of our knowledge, there is no simple way to induce neural networks to shift from waking mode to sleeping mode. Our best guess is that a whole group of neurons may be involved and that the process may develop over a period of time and a temporal sequence which are mostly unknown (3). 2) We previously reported that the sensory input - particularly the auditory one because is the one that remains continuously "open" - is part of the 
information used in dreams. Thus, it seems important to remark that more than half of the auditory cortical neurons recorded during sleep show a number of evoked discharges similar to that observed in wakefulness $(57.8 \%)$, and therefore they are continuously informing the brain. The auditory firing shifts that occur on passing to sleep augmenting (18.3\%) or diminishing (23.9\%) - are postulated as units locked to sleeprelated networks, whatever function they may play in sleep, perhaps related to auditory dream images (4). A similar number of auditory spontaneously firing units were recorded throughout the sleep-waking cycle, also suggesting a participation in dream images. 3) A number of auditory and visual neurons have demonstrated a firing phase-locking to the hippocampal theta rhythm, both during wakefulness and sleep. The theta rhythm may act as a temporal organizer of sensory information in visual and auditory systems in all behavioral states, adding a temporal dimension to the sensory processing $(5,6)$. This may be valid for the temporal organization of stored auditory information during dreams. 4) Finally, the sensory input that is admitted to "sculpt" the brain in a genetically determined manner, is active during sleep and - of uppermost importance - during early developmental stages, i.e., the first days and months of life when most of the time is spent in sleep (4).

Cesar Timo-Iaria: May I add to your last remarks that I have found (unpublished results) an intense glucose utilization in the entire auditory pathways, from the pons to the cerebral cortex during barbiturate anesthesia in experiments in which I measured glucose utilization by means of the ${ }^{14} \mathrm{C}-2$ deoxy-D-glucose method. I reasoned that the auditory system is probably the only open input system that is able to inform the brain about the presence of some dangerous animal (that may be a predator) during sleep and such role does not decay even during anesthesia; this system may wake up the brain if some menacing sound does occur in the neighborhood. But I think this function of the auditory system is not indiscriminate, inasmuch as a mother may continue to sleep when an airplane flies over her house but will be immediately awakened by her child's faintest vocalization. Vision is not able to do that because the eyes are closed and olfaction, the main sensory channel in rodents, is a weak detector of substances in the environment during sleep because sniffing does not occur in such a state, unless the animal is dreaming, a condition during which sniffing may be very active, although not directed at the environment, only at the dream. Also, may I add, we are presently mapping the theta rhythm in the olfactory system, having found that it is omnipresent, with the potentials being highly correlated all along the olfactory pathways, not to my surprise.

Florin Amzica: Slow-wave sleep is a state of compromise between active information processing and total annihilation of consciousness. This is achieved by means of a slow oscillation $(<1 \mathrm{~Hz})$ that has been described in intracellular recordings of cortical neurons (7), but equally in the EEG $(8,9)$ and EMG $(10)$ of humans. This cellular oscillation is marked by a continuous alternation of the membrane potential between two voltage levels: a depolarized one and a hyperpolarized one. The membrane depolarization lasts for about $0.4-0.8 \mathrm{~s}$, is due to synchronous synaptic activities in the cortical network (11), and consists mainly of excitatory and inhibitory postsynaptic potentials (7). The hyperpolarization is associated with silenced firing in the cortical network lasting generally 0.3 to $0.7 \mathrm{~s}(7,11,12)$, and is associated with a global removal of facilitation in the corticothalamic network (13) promoted by periodic variations of the extracellular calcium levels (14). Thus, the compromise I was referring to at the beginning might be an evolutionary adaptation between total rest (the hyperpolarizing phase of the slow oscillation) and some kind of 
preserved cortical activity, keeping the circuitry active so that it will be ready to work in the case of a significant awakening signal.

The depolarizing phase of the slow oscillation often generates activities in the 20-50$\mathrm{Hz}$ frequency band, which are associated with information processing. The result is that some repetitive mentation may occur during slow-wave sleep, and this in the absence of sensory input, since the thalamus as a relay station for the sensory afferents is hyperpolarized. It could be therefore hypothesized that this type of activity may represent slow-wave sleep dreaming. These dreams would be in contrast to those probably occurring during REM sleep, which seem to be more elaborate and continuous.

Cesar Timo-Iaria: I may have misunderstood you but it is hard to believe that during dreaming there is a total annihilation of consciousness, inasmuch as its conscious manifestation is the only one the dreamer is able to identify as a dream. We know we have dreamed exclusively through the conscious experience we had during sleep. Someone outside knows we are dreaming because of the movements we perform, that Aristotle knew very well to be the motor expression of the dream content. The conscious process, as I see it, is qualitatively the same during wakefulness and during the periods of oniric activity; it is less precise during the dreaming periods probably because in sleep the powerful activity of the activating systems, that characterizes the wakeful state and does provide the great accuracy of conscious identification, is very weak although not absent.

Luciano Ribeiro Pinto Jr.: Dreams derive from a manifestation of our limbic system, so we are inclined to suggest that dreams might be a physiological mechanism which periodically activates our reptilian or phylogenetically more ancient conscience.

Flavio Aloe: I do not quite agree that dream content is due in part to the activation of the limbic system which in turn creates the dreaming sensory phenomena.
Luciano Ribeiro Pinto Jr.: Neurobiological studies have shown that there is an activation of the limbic structure and a deactivation of part of the frontal cortex.

Flavio Aloe: I agree with you on that, but I believe the limbic system is co-activated along with other neural circuits as well.

Wayne Hening: Luciano, I think we can be too confident that we know all the mechanisms that generate any behavior, as the recent discovery of the hypocretin/orexin system and its potential relation to sleep/wake regulation suggests. It is true that we don't know what the rats are "experiencing", only that their brain seems to be active in a recognizable pattern.

Robert Vertes: I would somewhat agree with Luciano that dreams are a manifestation of the limbic system, with the caveat that they are not initiated at the level of the limbic system but at the level of the brainstem. In a position not too different from the original activation-synthesis hypothesis of Hobson and McCarley (15), I basically view dreams as the best fit to an undifferentiated activation of the brainstem on the forebrain during REM sleep. The brainstem globally activates the forebrain in REM which then sets in motion a competition among forebrain circuits, the final outcome of which determines the specific contents of dreams; in other words, the contents of stronger circuits prevail over those of weaker circuits and hence become incorporated into dreams. Various factors could affect the strength of circuits including recency, meaningfulness, redundancy, etc. A 100 or so years of psychoanalysis, however, cannot be discarded; dreams certainly have elements of the surreal, but nonetheless are strongly associated with our deepest needs and concerns. I would support, then, Hobson and McCarley's view (15) that the activation of the brainstem is essentially random and chaotic in REM, but the forebrain (or limbic system), at least partially, structures the chaos to the particular concerns of the dreamer. 
Cesar Timo-Iaria: May I comment upon some statements that deserve a few words of mine. Dr. Luciano's answer about the functions of sleep reminds me of Kleitman's hypothesis (16), according to which we should change the current concept that wakefulness is the basal state, which is periodically interrupted by sleep. It may be the other way around: sleep is the basal state, which is interrupted by wakefulness, so that an animal can explore the environment, eat, copulate, defend itself, etc. If this is true, then sleep may be understood much better than when wakefulness is taken as the basal state. Dr. Aloe said that sleep serves a multitude of functions, such as maturation of the nervous system, synaptic restoration, memory processes. Well, these are hypothetical statements so far; there may be something valid in such hypotheses but they cannot be taken as well-established knowledge. Among other reasons, since all mammals, birds, reptiles, amphibia, fishes and arthropods do sleep, as well demonstrated as far back as 2400 years ago by Aristotle, I stick to Kleitman's concept (16) that the basal state is sleep, not wakefulness.

Another concept that deserves consideration is Jouvet's hypothesis (2) according to which dreams are protective of the genetic integrity of our personality. This hypothesis seems science fiction to me. Let us not forget that, as shown by Aristotle and Darwin long ago, all mammals and birds do dream. "Our personality" seems to restrict dreaming to humans. Let me take advantage of this moment to recall that it was Aristotle who discovered eye movements, limb movements, lip movements, head movements, etc., as manifestations of dreaming, which he correctly related to the dream content. Some researchers in this field do not believe that movements exhibited during dreaming are directly linked to dream content, mainly because eye movements do not fit properly into what is being dreamed. That is a mistake, inasmuch as the powerful inhibition of mo- toneurons (due to hyperpolarization, as shown by Pompeian et al. (17) in 1967) does not allow a faithful reproduction of the programmed movements during dreaming, thus making any movement incomplete, uncoupled and sometimes not recognizable as part of a behavior. Electromyographic studies do disclose this functional uncoupling very well. The classic experiments performed in Jouvet's laboratory show without any question that when inhibition of the motoneurons is inactivated, a cat is able to execute coherent movements during dreaming (2).

We are very far from understanding the functions of dreaming but what a dream is can be tentatively explained more confidently. I see dreaming as the result of the conscious identification of memorized and combined information (whether it is actively retrieved by some neural system or is loosely released from mnemonic archives is hard to say but probably both mechanisms are involved). Much the same happens when we think during wakefulness. We all know that the traffic of information in the nervous system is astoundingly intense. Any information has to be decoded and identified before it can be used, after which it can be identified. Fortunately, most information is identified by unconscious processes, otherwise we would be consciously monitoring all the time our blood pressure levels, glycemia, $\mathrm{pH}$ in the stomach and intestines, $\mathrm{pO}_{2}$ and $\mathrm{pCO}_{2}$, plasma testosterone and estrogen concentration, circulating antibodies, etc. Worse than that, we would be monitoring what is happening in every microcircuit in the entire nervous system. Luria's (18) famous patient was aware of what a tragedy bringing into consciousness so much information can be; he could not pay attention to anything without a tremendous effort to select what attention should be directed at.

Selecting a very small amount of information at any moment to be identified by the conscious process is absolutely necessary for attention. An analogy may be worked out 
by comparing what is going on in the nervous system and radiation delivered in space by all the heavenly bodies. Through vision we are able to identify only a very narrow band of radiation, between near 400 and 800 $\mathrm{nm}$ in wavelength; yet, we have the impression that opening the eyes is enough to disclose the entire universe to our understanding but in fact this is only an extremely small portion of the whole spectrum of radiation, which spans from radiofrequencies to gamma rays. When we see pictures of the sky in the $\mathrm{X}$-ray or in the infrared bands we can appreciate how little is shown by (visible) light alone. By the same token, consciousness is the "visible" portion of the spectrum of identification of information in the nervous system; most of the information that is running is not identified through the conscious process. When we dream we certainly do identify through the conscious process only a small fraction of what is going on in the brain, just as during wakefulness.

Luiz Menna-Barreto: Sleep and wakefulness are both normal states, and the basal state should thus be understood as the oscillation between these expressions. Cycling is perhaps the adaptive link to our environment.

Ricardo A. Velluti: The enormous amount of changes in whole body physiology must lead us to consider some general sleep actions. I agree that cycling is an adaptive link to the environment and the body.

Wayne Hening: The most exciting report that I have heard lately is the one documenting a pattern of neural activity during REM sleep in rats similar to that which is seen during active maze running (19).

Flavio Aloe: Would you recall what circuits displayed this interesting pattern you mentioned in rats?

Wayne Hening: The neurons monitored were in the hippocampus. I guess the general suggestion is that there can be an actual reprise of experienced neural activity, even at the cellular level during sleep. I have not seen the report itself, only a journalist's article in the New York Times. It was in Science (19), one of the major journals.

Luciano Ribeiro Pinto Jr.: I would like to know what role sawtooth waves could play in dreams.

Flavio Aloe: Yes, to start with, I think sawtooth waves are a phasic element of REM sleep.

Luiz Menna-Barreto: I find it very difficult to generalize when defining CNS functions - perhaps sleep is a good term for mammals, but one that loses precision outside our "big family". Nevertheless, dolphins and their alternating hemispheres are one of the nice examples of cycling as a key phenomenon in CNS evolution, don't you agree?

Ricardo A. Velluti: We are far away from such knowledge. I believe that, at this moment, to compare or to link a group of waves with an oniric activity is impossible.

Flavio Aloe: Menna, the sleep-to-wakefulness-to-sleep (SWS) oscillation is a key feature, but dolphins for instance are able to present hemispheric sleep to remain partially awake and keep away from predators.

Robert Vertes: I am a little more familiar with this work. An original study by Constantine Pavlides and Jonathan Winson (20) showed that specific patterns of pyramidal cell discharge of the hippocampus during waking are repeated in REM sleep; that is, hippocampal place cells exposed to their place fields during waking were much more likely to exhibit similar patterns of discharge in REM compared to place cells not exposed to their place fields in waking. Bruce McNaughton and colleagues $(19,21)$ subsequently described virtually identical findings with the important difference that the hippocampal "replay" occurred in SWS and not in REM sleep. Finally, in the study referred to here, Louie and Wilson (22) reported that fairly long sequences (10 seconds to a minute) of hippocampal ensemble activity seen during wakeful maze learning 
were repeated in subsequent REM sleep. The authors of these various reports argue that the reactivation of patterns of waking activity in sleep (SWS or REM) serves to process or consolidate these experiences, i.e., sleep is critical for memory consolidation. I not only disagree with the interpretation of these findings, but with the general notion that REM sleep is vital for memory consolidation. Regarding the replay of patterns of waking activity in sleep, I would hold that this by no means indicates that the replay serves to strengthen memory traces of waking in sleep; the replay may merely represent the reoccurrence of daytime images/ experiences in dreams. As Dr. Timo-Iaria aptly points out (see below), $80 \%$ of human dreams may be directly linked to experiences of the day before. Like most (or virtually all) material of dreams, these traces would not be permanently stored or consolidated into memory. On the general issue of memory consolidation in REM sleep, we $(23,24)$ have recently argued against it, pointing out that: 1) early reports examining the effects of REM deprivation on memory in animals were about equally divided in showing that REM deprivation does, or does not, disrupt learning/memory, but studies supporting a relationship between REM sleep and memory have been strongly criticized based on the confounding effects of very stressful REM deprivation techniques (i.e., the inverted flower pot technique); 2) the three major classes of antidepressant drugs, monoamine oxidase inhibitors, tricyclic antidepressants and selective serotonin reuptake inhibitors, profoundly suppress (or eliminate) REM sleep but do not disrupt learning/ memory, and 3) recent functional imaging studies in humans have revealed patterns of brain activity in REM sleep that are inconsistent with memory consolidation in REM; for instance, as others have pointed out here, there is a marked reduction in activity in the frontal/prefrontal cortex in REM sleep. In effect, activity within memory processing systems of the brain appears to be attenuated in REM sleep, leading Barbara Jones (25) to recently observe that this is consistent with "an attenuation of processes important in episodic and working memory and perhaps explaining why, unless awakened from a dream, a sleeping person has no memory of the dream". It would seem that most proponents of the memory consolidation hypothesis in REM would agree that waking experiences are not faithfully reproduced in dreams or committed to memory through dreams. In a twist of logic, however, that we find difficult to understand, it appears that proponents of the consolidation hypothesis seem willing to acknowledge that conscious material of REM (dreams) is not stored in REM sleep, while at the same time holding that material that never reaches dream consciousness (whatever its nature) is somehow magically processed and consolidated in REM sleep. In summary, I would hold that there is no compelling evidence that REM sleep serves any role in the processing and consolidation of memory.

Cesar Timo-Iaria: I have not read yet Louie and Wilson's paper (22) on the correlation between dreaming and some manifestations of the behavior displayed by rats during active maze running but Wilson himself and McNaughton (21) had already discovered in 1994 that some hippocampal neurons that are very active during wakefulness are also active during desynchronized sleep, displaying a similar functional pattern in both circumstances. The similarities between wakefulness and dreaming can also be studied in depth through theta waves. Theta rhythm is a good marker for the electrophysiological analysis of both wakefulness and desynchronized sleep in the nervous system of rodents. We have subjected theta rhythm to several types of analysis and found that it usually unveils episodes of arousal and dreaming activity by increasing the frequency and voltage of the potentials. When oniric movements are intense, for instance, 
theta waves are substituted for by desynchronization, resembling the potentials that characterize oniric activity in humans. Desynchronization is well known to characterize attentive wakefulness in humans, which may indicate that in rats desynchronization replaces theta waves when a high degree of attention is being produced by the brain.

Preceding an oniric episode, theta waves display a synchrony of voltage among the hippocampus and somesthetic cortical areas but when a dream begins they become asynchronic; frequency, which varies preceding the oniric episode, increases during dreaming and then becomes similar in all leads. Such oscillations of voltage span from 0.5 to $4 \mathrm{~Hz}$ and theta frequency may increase up to $11 \mathrm{~Hz}$; therefore, theta waves display a very interesting kind of AM and FM modulation during desynchronized sleep.

Serious studies on dreaming provide more and more support to those who do not agree with the psychoanalytic interpretation of dreams and agree with the incredible (and unknown) paper named Statistics of Dreams, published by Ann Calkins in 1876, in which she states that nearly $80 \%$ of human dreams are directly linked to the experience the day before. I guess Ann Calkins was the one who invented the technique of arousing a person who is moving the eyes, the fingers, the legs and the lips while sleeping, in order to learn the dream content reported by the dreamer, and she made a good use of it. By the way, I learned about Calkins' findings in an Italian book published in 1899 in Turin, written by an important neuropsychiatrist, Sancto de Santis, who described dementia praecox. This book lists nothing less than 323 references on dreaming (in 1889; most sleep specialists think that such studies began in the early 1950's!). Much knowledge existed related to dreaming during the last quarter of the 19th century but psychoanalysis managed to bury it during the 20th century. Loose reading of ancient literature has also contributed to such nice work having disappeared from science. These facts should induce people to read ancient literature about everything. There is a marvelous treasure waiting for us to be dug out. All people involved in sleep research should read at least Aristotle's book on sleep and dreams.

Flavio Aloe: Whatever the minute precision of Wilson and McNaughton's paper (21) at this point in time, it is my impression that behaviors or a wider definition of them can occur in a striking similar fashion both during wakefulness and during REM sleep. What are everybody's thoughts?

Luciano Ribeiro Pinto Jr.: What's your opinion about some clinical aspects involved in dreams, such as epic dreams, nightmares and status dissociatus?

Wayne Hening: Certainly, the vivid enactments of REM sleep behavior disorder suggest that dream content can correspond to normal, if fantastic, actions, since they are acted out.

Ricardo A. Velluti: Cesar, I agree with you and Ann Calkins. Most of the contents in our dreams have to be previously present in our sensory systems.

Luiz Menna-Barreto: What about fantastic associations?

Ricardo A. Velluti: The dreams are a product of our brain depending on its genetically determined structure (and perhaps information). That is also why a depressed patient has depressed dreams or some brain lesion has special dream contents (prosopagnostic patients).

Luiz Menna-Barreto: Random sweeping of information seems to me a better description of dream content, not necessarily linked to a specific sensory experience.

Cesar Timo-Iaria: The raw material of dreams is, without any doubt, memorized information, as it is for wakeful thought. Bits of mnemonic information can be combined in a great many ways (whose rules we do not know yet). This concept explains any kind of dream, be it a common visual scene, an epic dream, a nightmare or any impossible dream, 
just as it is possible to combine evoked memorized information and invent a joke, a song or a scientific theory, as well as picturing the queen of England dancing charleston or rock and roll with the pope... By combining retrieved or released memorized information it is possible to build up any pattern. Of course, the brain of the future primate species (within 2, 3 and more millions of years) will be able to perform such combinations in much more complex and powerful ways than our brain can. I guess they will experience mental images we cannot presently foresee.

Flavio Aloe: Your model is certainly impressive, but one particular situation sort of challenges it, the lucid dreaming of some individuals who can actively control the dream they are having.

Cesar Timo-Iaria: You picked up a very interesting and impressive phenomenon, which, I guess, fits well into my model. Probably, the lucid dreamer is in a state in between wakefulness and desynchronized sleep, which renders it possible for him to drive the dream (although not as perfectly as thoughts and imagery during wakefulness).

Flavio Aloe: Most certainly. Then, how do the dreams of congenitally blind individuals fit into your model?

Cesar Timo-Iaria: Well, this is a difficult question to deal with. Yet, let me try to give you a convincing answer... Humans are eminently visual animals, as are other primates and most birds. It means that other, non-visual sensory information may start a behavior that is intended to switch attention to the visual system and then visual information is the one that drives the decision to produce a convenient behavior in response to non-visual information. We all have the trivial experience that when we hear someone knocking on a door we immediately turn the head to scan the visual scene, despite the original information being an auditory one. It is led by visual information that we walk precisely to the door to open it. Rats, on the other hand, try to smell anything they look at, simply because they are primarily macrosmatic animals. The systems that generate behaviors are wired in such a way that the prevailing sensory channel in a particular species does, most probably, have direct access to the effector pathways. I am quite convinced that other sensory channels (as well as mnemonic and voluntary generators of behavior) utilize the prevailing pathways to produce a certain behavior; such pathways appeared in primitive vertebrates and were not discarded by nature when birds and mammals developed. We all know that the old rhinencephalon, primarily related to olfaction, is the core of such pathways, because olfaction has been the most important sensory channel in vertebrates from their very appearance on earth and subsequently guided the development of most behavioral systems. This marvelous hodological arrangement has probably led MacLean (26) to invent the "limbic system", that has evolved to become a fashion and a fiction, according to the great neuroanatomist Brodal, inasmuch as the entire nervous system is limbic... I agree with him!

In our experiments on wakefulness and dreaming activity in rats we routinely record electrical potentials from many muscles, as well as from the electrical field produced around the eyes by ocular movements. During wakefulness any kind of sensory stimulation causes an immediate activation of the muscles that move the vibrissae and rostrum. We have found also that $48.9 \%$ of the dreams experienced by rats are expressed as rostrum and vibrissa movements; in humans, that are eminently visual animals, nearly $100 \%$ of the dreams are behaviorally expressed as eye movements. People who have become blind after acquiring a visual "universe" are known to dream visual dreams, with plenty of figures and visual scenes. Congenitally blind people cannot dream of figures and visual scenes because such patterns are not in their memory but they may have visual sensations accompanying auditory and tactile dreams. 
I see it as a switching of other kinds of information to vision; the visual circuits are almost absent in congenitally blind people, but the visual cortex, however deformed, does exist and is probably invaded by auditory and other kinds of sensory projections; at least in experimental animals such invasion has been repeatedly demonstrated.

There is a fascinating phenomenon that may be related to this mechanism, discovered by Hughlings Jackson in epileptic patients during the last quarter of the 19th century and extended during the first half of the 20th century by Forster and Penfield through electrical stimulation of the cerebral cortex of people subjected to several kinds of neurosurgical procedures. Jackson discovered that in some epileptic patients whose aura was experienced just as flashes of bright and white light the epileptic lesion was in the occipital cortex (areas 17 and 18). Only in patients whose lesion was located in the posterior portion of the parietal and temporal lobes did the aura consist of visual scenes and organized figures. Presently it is well known that in humans and other primates there are 32 visual cortical areas; area 17 only receives faithful information about the position of every light signal arising from the retina but this is not enough to produce real vision; it transmits such primitive information to the other areas, which depend on comparing it with memorized information to assemble the real external scene. The other, more frontally located areas are the source of real visual images with a content. Visual dreaming in congenitally blind people does certainly not contain visual scenery but may contain flashes of light. Electrical stimulation of these same areas yields similar results.

Now, may I comment on Dr. Velluti's hypothesis about the functions of the theta rhythm. I think theta waves are in fact a command signal whose function is to multiplex all the circuits that are involved at a certain moment in producing a certain behavior, be it a wakeful one, be it a dream. In our laboratory it has been shown that theta waves may be closely coupled in many central sites during attention and during dreaming. Among the multiplexing functions of these waves, I agree that there may be the addition of a temporal dimension to the sensory processing, which is certainly necessary for the timing of information that creates any behavior, including thinking and dreaming.

\section{Acknowledgments}

The authors thank Dr. Claudio Da Cunha for organizing the chat series "The Brain Decade in Debate" and CNPq-Prossiga for providing the chat web room.

\section{References}

1. Aserinsky E \& Kleitman N (1953). Regularly occurring periods of eye motility, and concomitant phenomena, during sleep. Science, 118: 273-274.

2. J ouvet M (1988). The regulation of paradoxical sleep by the hypothalamo-hypophysis. Archives Italiennes de Biologie, 126: 259-274.

3. Velluti RA, Pedemonte $M \&$ Peña J L (2000). Reciprocal actions between sensory signals and sleep. Biological Signals and Receptors, 9: 297-308.
4. Peña J L, Pérez-Perera $L$, Bouvier $M \&$ Velluti RA (1999). Sleep and wakefulness modulation of the neuronal firing in the auditory cortex of the guinea-pig. Brain Research, 816: 463-470.

5. Gambini J P, Velluti RA \& Pedemonte M (2001). Functional correlation between hippocampal theta rhythm and lateral geniculate neurons in sleep and waking. Neuroscience Letters (in press).

6. Pedemonte $M$, Pérez-Perera $L$, Peña $L L \&$ Velluti RA (2001). Auditory processing dur- ing sleep. Correlation of cortical unitary activity with hippocampal theta rhythm. Sleep Research (in press).

7. Steriade $M$, Nuñez $A \&$ Amzica $F$ (1993). A novel slow $(<1 \mathrm{~Hz})$ oscillation of neocortical neurons in vivo: depolarizing and hyperpolarizing components. J ournal of Neuroscience, 13: 3252-3265.

8. Achemann P \& Borbély AA (1997). Lowfrequency $(<1 \mathrm{~Hz})$ oscillations in the human sleep EEG. Neuroscience, 81: 213222. 
9. Amzica $F \&$ Steriade M (1997). The Kcomplex: Its slow $(<1 \mathrm{~Hz})$ rhythmicity and relation to delta waves. Neurology, 49: 952-959.

10. Simon NR, Manshanden I \& Lopes da Silva FH (2000). A MEG study of sleep. Brain Research, 860: 64-76.

11. Amzica F \& Steriade M (1995). Short- and long-range neuronal synchronization of the slow $(<1 \mathrm{~Hz})$ cortical oscillation. J ournal of Neurophysiology, 75: 20-38.

12. Contreras $D \&$ Steriade M (1995). Cellular basis of EEG slow rhythms: a study of dynamic corticothalamic relationships. J ournal of Neuroscience, 15: 604-622.

13. Contreras D, Timofeev $I \&$ Steriade $M$ (1996). Mechanisms of long-lasting hyperpolarizations underlying slow sleep oscillations in cat corticothalamic networks. J oumal of Physiology, 494: 251-264.

14. Massimini $M \&$ Amzica F (2001). Extracellular calcium fluctuations and intracellular potentials in the cortex during the slow sleep oscillations. J oumal of Neurophysiology, 85: 1346-1350.

15. Hobson JA \& McCarley RW (1977). The brain as a dream-state generator: An activation-synthesis hypothesis of the dream process. American J ournal of Psychology, 134: 1335-1348.

16. Kleitman N (1974). Leaming and sleep theory and practice of hypnopaedia Rubin F. J oumal of Nervous and Mental Disease, 159: 293-294.

17. Pompeian O, Carli G \& Kawamura H (1967). Transmission of sensory information through ascending spinal hindlimb pathways during sleep and wakefulness. Archives Italiennes de Biologie, 105: 529531.

18. Luria AR, Homskaya ED, Blinkov SM \& Critchle M (1967). Impaired selectivity of mental processes in association with a lesion of frontal lobe. Neuropsychologia, 5: 105-110.

19. Skaggs WE \& MCNaughton BL (1996). Replay of neuronal firing sequences in rat hippocampus during sleep following spatial experience. Science, 271: 1870-1873.

20. Pavlides C \& Winson J (1989). Influences of hippocampal place cell firing in the awake state on the activity of these cells during subsequent sleep episodes. J ournal of Neuroscience, 9: 2907-2918.

21. Wilson MA \& McNaughton BL (1994). Reactivation of hippocampal ensemble memories during sleep. Science, 265: 676-679.

22. Louie K \& Wilson MA (2001). Temporally structured replay of awake hippocampal ensemble activity during rapid eye movement sleep. Neuron, 29: 145-156.

23. Vertes RP \& Eastman KE (2000). The case against memory consolidation in REM sleep. Behavioral and Brain Sciences, 23: 867-876.

24. Vertes RP \& Eastman KE (2000). REM sleep is not committed to memory. Behavioral and Brain Sciences, 23: 10571063.

25. J ones BE (1998). The neural basis of consciousness across the sleep-waking cycle. In: J asper HH, Descaries L, Castellucci VF \& Rossignol S (Editors), Consciousness: At the Frontiers of Neuroscience, Advances in Neurology. Vol. 77. Lippincott-Raven Publishers, Philadelphia, PA, USA.

26. MacLean PD (1954). The limbic system and its hippocampal formation - Studies in animals and their possible application to man. J oumal of Neurosurgery, 11: 29-44. 